\title{
Conditioned withdrawal signs elicited by contextual cues for morphine administration
}

\author{
MARVIN D. KRANK \\ Mount Allison University, Sackville, New Brunswick, Canada \\ and \\ WENDY L. PERKINS \\ University of Waterloo, Waterloo, Ontario, Canada
}

\begin{abstract}
Pavlovian conditioning theories of drug dependence argue that drug-or withdrawal-associated cues elicit motivational conditioned responses that enhance the likelihood or intensity of drugtaking behavior. The present experiment assessed whether drug-associated cues can elicit a conditioned withdrawal reaction in rats. In support of previous research, we found clear evidence for conditioned withdrawal. When tested without morphine in a distinctive environment, animals that had received morphine paired with that environment showed more pronounced signs of withdrawal than did animals that had received equivalent doses of morphine unpaired with the test environment. Despite differential levels of withdrawal reactions, consumption of the potent opiate etonitazene in the test environment was not influenced by conditioning. Prior morphine experience did increase both the rate of barpressing for and the level of consumption of oral etonitazene compared with saline control levels. Performance in paired animals, however, was not superior to that in unpaired animals. Although clearly demonstrating conditioned withdrawal, these observations question the importance of withdrawal in the acquisition of oral selfadministration of an opiate.
\end{abstract}

Several theories of addictive behavior have the core assumption that Pavlovian drug conditioning motivates drugtaking behavior (Poulos, Hinson, \& Siegel, 1981; Siegel, 1979, 1983; Stewart, de Wit, \& Eikelboom, 1984; Wikler, 1973). The central basis for these associative theories is that animals exposed to contingencies between environmental conditioned stimuli (CSs) and drug administration unconditional stimuli (USs) acquire conditioned responses (CRs; see Eikelboom \& Stewart, 1982; Siegel, 1989; Siegel, Krank, \& Hinson, 1987). Subsequent exposure to drug-associated CSs stimulates powerful behavioral and physiological reactions in anticipation of drug effects that may mediate drug-taking behavior.

Conditioning theorists have proposed three distinct mechanisms by which CSs may enhance drug-taking behavior: conditioned incentive, conditioned withdrawal, and selective tolerance. Conditioned incentive theorists argue that the drug CS activates a positive incentive-state CR that motivates consumption to regain the appetitive effects of the drug (Stewart, de Wit, \& Eikelboom, 1984). By contrast, conditioned withdrawal theorists postulate that the drug CS elicits a withdrawal syndrome that moti-

\footnotetext{
We would like to thank Susan O'Neill for her assistance in the collection and analysis of data described here. This research was supported by a grant from the National Science and Engineering Research Council of Canada to the first author. Our thanks are extended to the Bureau of Drug Research, Health, and Welfare of Canada for the donation of etonitazene for this research. Requests for reprints should be addressed to M. D. Krank, Department of Psychology, Mount Allison University, Sackville, NB, Canada E0A 3C0.
}

vates drug-taking to alleviate this aversive condition (Poulos et al., 1981; Siegel, 1979; Wikler, 1973). A third potential mechanism derives from recent suggestions that conditioned tolerance to the drug's aversive effects may be more pronounced than its appetitive effects (Krank, 1989; Tabakoff \& Hoffman, 1988). Because such conditioning would be expected to enhance the drug's net reward value by reducing its negative effects more than its positive effects, selective tolerance should have motivational consequences.

Despite rich theoretical differences among these three distinct mechanisms, most studies have provided only general support for the role of Pavlovian drug conditioning in drug-taking behavior. Such evidence comes from studies showing that the presence of drug CSs for ethanol (Krank, 1989; Krank, O’Neill, \& Brake, 1989), cocaine (de Wit \& Stewart, 1981), and morphine (Hinson, Poulos, Thomas, \& Cappell, 1986) increase drug-taking behavior. In addition, exposure to CSs conditioned with an ethanol US reduces the resumption of ethanol self-administration after a period of abstinence (extinction; Krank \& Wall, 1990). However, such simple demonstrations of drug conditioning effects on drug-taking behavior do not serve to differentiate the three proposed mechanisms. Nevertheless, the three approaches make differential predictions that should be detectable by behavioral tests (see, e.g., Falls \& Kelsey, 1989; Stewart, de Wit, \& Eikelboom, 1984).

The present study was designed to test whether a CS conditioned with an opiate as the US has motivational properties that are related to conditioned withdrawal, as 
expected by Siegel's compensatory response theory (Hinson \& Siegel, 1980; Poulos et al., 1981; Siegel, 1979, 1983). According to Siegel and his associates, drug CRs are often opposite or compensatory to the direct effects of the drug (see Krank, 1987; Krank, Hinson, \& Siegel, 1981). When the drug is administered, the compensatory CRs that are elicited are expected to contribute to tolerance. However, when the drug is not administered, such compensatory CRs are expected to produce an aversive withdrawal state that motivates consumption of the drug to relieve the discomfort. In the present studies, we tested whether a CS conditioned with opiate administration as the US elicits a withdrawal CR and whether this CR correlates with opiate self-administration. Such a correlation is predicted by theories attributing a motivational role to Pavlovian conditioned withdrawal.

Several previous studies have looked for conditioned withdrawal with opiates as the US. Kelsey and his associates (Falls \& Kelsey, 1989; Kelsey, Aranow, \& Mathews, 1990 ) report that conditioning with an opiate US increases the magnitude of several withdrawal signs in the rat. Other investigators (Sobrero \& Bouton, 1989; Zellner, Dacanay, \& Riley, 1984) have not observed conditioned withdrawal under conditions where such conditioning might be expected. The studies that found no evidence for conditioned withdrawal differed from Kelsey and associates' studies in terms of type of CS and the timing of opiate administrations. As in Kelsey and associates' studies, the environmental cues and timing of the opiate injections we used here approximated opiate conditioning studies that have revealed conditioned tolerance (Siegel, Hinson, \& Krank, 1978) and compensatory CRs (Krank, 1987; Krank et al., 1981). Although the role of compensatory CRs in tolerance has been challenged (Baker \& Tiffany, 1985; Tiffany, Petrie, Baker, \& Dahl, 1983; but see Krank, 1987), Siegel's $(1979,1983)$ proposed relationship between conditioned tolerance and conditioned withdrawal suggests that the use of these parameters should increase the likelihood of detecting conditioned withdrawal.

A few previous studies have reported that conditioning with opiates as the US increases opiate consumption (Hinson et al., 1986; Thompson \& Ostlund, 1965). Strong evidence for the role of Pavlovian conditioning in opiate consumption was found in a transfer of training design (Trapold \& Overmier, 1972) used by Hinson and his associates (1986). They reported that response-independent signals for morphine increase morphine consumption in animals with extensive drug-taking experience. They did not, however, report evidence of conditioned withdrawal; thus, any relationship between withdrawal and opiate consumption was unknown. That relationship is the central concern of this study.

The likelihood of detecting opiate conditioning effects on drug-taking behavior in the present study was enhanced by assessing Pavlovian transfer effects on operant selfadministration of the opiate etonitazene. Etonitazene is relatively tasteless at effective dosages, is absorbed well by the gastrointestinal tract, and is effectively self- administered orally. Specifically, animals will respond selectively for and consume pharmacological doses of etonitazene solutions (Carroll \& Meisch, 1979; Wikler, Martin, Pescor, \& Eades, 1963). Thus, we were able to study opiate self-administration under conditions similar to those that we have used successfully with ethanol (Krank, 1989; Krank et al., 1989; Krank \& Wall, 1990). We expected that a CS conditioned with an opiate US would elicit withdrawal reactions and enhance etonitazene self-administration.

\section{METHOD}

\section{Subjects}

The subjects were 48 experimentally naive, male Sprague-Dawley (CD) rats from Charles River Canada in St. Constant, Quebec. The animals, housed individually in stainless steel hanging cages, were on a 12:12-h light:dark cycle (0900-2100 h light). The mean animal weight at the start of the experiment was $253.5 \mathrm{~g}(S D=11.6)$. Food and water were freely available in the animal's home cage, except as described in the Procedure section.

\section{Apparatus}

Four Lafayette Instruments (Model 80001) operant conditioning chambers were used. All chambers were equipped with two retractable levers on each side of the front panel of the chamber, two translucent cue lights above the lever locations on each side of the front panel of the chamber, and a liquid reinforcement dispenser located in the center of the front panel between the two levers. Each operant conditioning chamber was enclosed in an isolation box $(60.0$ $\times 40.0 \times 44.0 \mathrm{~cm}$ ), with a constant masking noise of $72 \mathrm{~dB}$ (SPL, A scale) from a ventilating fan and illumination from a $25-\mathrm{W}$ red houselight. The operant chambers were controlled by an Apple IIe computer via a Lafayette Instruments interface.

During videotaping sessions, four closed circuit Panasonic video cameras (Model WV 1500) recorded the rat's behavior in each operant chamber from about $30 \mathrm{~cm}$ above at a distance of $90 \mathrm{~cm}$ from the front of the chamber. For these sessions and for two sessions prior to the withdrawal test, the room containing the chambers was dark and the front door of each isolation chamber remained open. Light for filming was provided by the red houselight for the chamber. To improve visibility from the back of the chamber, a mirror $(30.5 \times 30.5 \mathrm{~cm})$ was slanted against the back wall of each isolation chamber. This provided a reverse image of the animal from the back.

\section{Procedure}

Habituation and barpress training. Initial operant training took place on the left lever (the right lever remained retracted throughout the experiment). After shaping, the animals were gradually shifted from an FR 1 to progressively more stringent VI schedules. Stable operant performance was established on a VI20-sec schedule and maintained for eight sessions. The reinforcement was a $0.1-\mathrm{ml}$ drop of a $0.1 \%$ saccharin solution. A 23-h/day water-deprivation schedule was in effect during the initial nondrug training. Free food and water were available in the home cage throughout the remainder of the experiment. All sessions lasted $50 \mathrm{~min}$ and were separated by $48-72 \mathrm{~h}$.

Morphine training. After habituation and initial barpress training, three groups of animals, matched for their terminal barpress rates (each $n=16$ ), received differential conditioning experience with a distinctive location (the operant chamber with the lever retracted) and a drug injection (either morphine or saline). All injections were s.c. in the dorsal area of the neck. Group paired animals were exposed to the operant chamber in conjunction with 
ascending doses of morphine sulfate on 10 occasions $(2 \times 10 \mathrm{mg} / \mathrm{kg}$, $4 \times 20 \mathrm{mg} / \mathrm{kg}$, and $4 \times 40 \mathrm{mg} / \mathrm{kg}$ ). These injections occurred $15 \mathrm{~min}$ after the animal had been placed in the chamber. On days preceding the operant chamber exposure, the animals were injected with saline in their home cages. Group unpaired animals received the same number and dose of morphine and saline injections, but for these animals, saline injections were given in the operant chamber and morphine injections were given in the home cage $24 \mathrm{~h}$ prior to their exposure to the operant chamber. Finally, group control animals received saline injections both during exposure to the operant chamber and in the home cage. Exposures to the operant chambers were separated by $48 \mathrm{~h}$ ( 7 injections) or $72 \mathrm{~h}$ ( 3 injections). All operant chamber exposures lasted $50 \mathrm{~min}$

Withdrawal test. Four days after its 10th morphine injection, each animal was tested in the operant chamber for $45 \mathrm{~min}$. Fifteen minutes into this session, each animal was given an injection of naloxone $(2 \mathrm{mg} / \mathrm{kg}$, i.p.). These sessions were videotaped and subsequently analyzed for various categories of activities indicating visible signs of withdrawal (see Table 1).

Videotapes of animals during the 45-min withdrawal test were scored for a variety of behavioral indices that have been reported in the literature as indications of withdrawal (Blasig, Herz, \& Reingold, 1973; Mucha, Kalant, \& Linseman, 1979; Wei, 1973). The scoring intervals were divided into three 15-min blocks: one prenaloxone and two postnaloxone. The measures scored were as follows: circles, completing one rotation; jumps, all four paws leaving the ground; darts, rapid movement from one part of the chamber to another; chews, biting of the paws; mouthing, masticating various surfaces in the chamber; rearing time, time spent with forepaws elevated; ear wipes, scratching at the ears; head shakes, shudder movement restricted to the head; wet dog shakes, full body shake; twitches, brief full body shudder; and paw tremors, visible shaking of the paws. Higher scores on each of these measures are taken as indices of withdrawal, except that decreased rearing time is an indication of withdrawal (Mucha et al., 1979). Tapes were scored by two observers, each of whom was blind to the group assignment of individual animals. Overall interrater reliability was .89 .

Reminder training. After the withdrawal test, the animals were allowed to rest and recover for a period of 26 days. All animals were then given two morphine reminder conditioning trials under the same group and temporal contingencies as in the conditioning phase (morphine dose $=20 \mathrm{mg} / \mathrm{kg}$ ). For each reminder, paired animals received morphine in the operant chamber, whereas unpaired and control animals received saline injections. Unpaired animals

Table 1

Mean Withdrawal Scores for Each of the Three Experimental Conditions for the 15-min Prenaloxone Scoring Interval

\begin{tabular}{|c|c|c|c|c|c|c|}
\hline \multirow{3}{*}{$\begin{array}{l}\text { Withdrawal } \\
\text { Measure }\end{array}$} & \multicolumn{6}{|c|}{ Group } \\
\hline & \multicolumn{2}{|c|}{ Paired } & \multicolumn{2}{|c|}{ Unpaired } & \multicolumn{2}{|c|}{ Control } \\
\hline & $M$ & $S E M$ & $M$ & $S E M$ & $M$ & $S E M$ \\
\hline Rearing time & $67.4 * \dagger$ & 7.15 & 87.8 & 6.66 & 88.2 & $5 . \overline{71}$ \\
\hline Wet dog shakes & $2.1 \dagger$ & 0.40 & 0.5 & 0.18 & 1.1 & 0.41 \\
\hline Twitches & $4.0^{*}+$ & 1.46 & 0.4 & 0.27 & 2.4 & 0.80 \\
\hline Ear wipes & $2.3 * \dagger$ & 1.09 & 0.2 & 0.14 & 0.3 & 0.18 \\
\hline Head shakes & $0.8 * \dagger$ & 0.28 & 0.0 & 0.00 & 0.1 & 0.06 \\
\hline Paw tremors & $1.3^{*}$ & 0.33 & 0.8 & 0.27 & 0.5 & 0.13 \\
\hline Circles & 1.2 & 0.50 & 0.3 & 0.15 & 0.3 & 0.12 \\
\hline Jumps & 2.8 & 0.90 & 1.8 & 0.62 & 4.8 & 1.39 \\
\hline Darts & 6.6 & 2.01 & $2.0^{*}$ & 0.64 & 10.2 & 2.76 \\
\hline Chews & 12.0 & 2.92 & $6.6^{*}$ & 1.56 & 16.9 & 5.56 \\
\hline Mouthing & 1.6 & 0.82 & 2.1 & 0.95 & 0.1 & 0.12 \\
\hline
\end{tabular}

*The value differs significantly from the corresponding control value. $\dagger$ The paired value differs significantly from the corresponding unpaired value. received morphine $24 \mathrm{~h}$ prior to their exposure to the operant chamber, whereas paired and control animals received saline.

Etonitazene self-administration. Four days after the second reminder conditioning session, all animals were returned to the operant chamber with the left bar in place. Barpresses were reinforced on a VI20-sec schedule. The reinforcement consisted of a $0.1-\mathrm{ml}$ drop of sweetened etonitazene $(5 \mu \mathrm{g} / \mathrm{ml}$, diluted in $.1 \% \mathrm{w} / \mathrm{v} \mathrm{sac}$ charin flavored solution of distilled water). The number of barpresses, reinforcements, and volume of solution consumed were recorded over six 50-min sessions. Sessions were separated by $24 \mathrm{~h}$.

\section{RESULTS}

\section{Habituation and Barpress Training}

Performance on the final two VI20-sec operant sessions did not differ among the three groups. One-way betweensubject analysis of variance (ANOVA) demonstrated no statistically significant differences between the groups on the number of barpresses, the number of saccharin reinforcements, or the weight of the animals. The means and SEMs on these three measures were, respectively, as follows: $719 \pm 64.8,125 \pm 2.86$, and $437 \pm 12.8$, for group paired; $718 \pm 62.1,123 \pm 2.37$, and $452 \pm 9.3$, for group unpaired; and $716 \pm 65.7,124 \pm 3.30$, and $434 \pm 10.9$, for group control.

\section{Morphine Training}

The only measure recorded during morphine training was the weight of the subjects. Weight changes between the initial and final morphine-injection day differed significantly between the groups $[F(2,45)=7.45, p=$ $.002]$. Newman-Keul's post hoc comparisons revealed that the two drug-treated groups, both unpaired and paired, differed from the drug-naive control group (both $p s<.01)$. Both drug groups had either not gained any weight over the morphine training phase or had actually lost weight. Control-group animals, on the other hand, had consistently gained weight during this period (mean $\pm S E M, 47.3 \pm 5.6$ ). Although unpaired animals lost more weight (mean $\pm S E M,-24.7 \pm 12.6$ ) as a function of morphine administration than did paired animals (mean $\pm S E M,-5.3 \pm 8.2$ ), this difference was not significant.

\section{Withdrawal Test}

Initially, an overall multivariate analysis of variance (MANOVA) on the 11 individual withdrawal measures was conducted using group as a between-subject factor and the scoring interval (pre, post 1 and post 2) as a within-subject factor. Not surprisingly, there was a significant interval effect [Wilks's lambda $=0.114$, $F(2,44)=170.14, p<.0001]$, indicating that the total number of behavioral responses declined over the three intervals. More relevant to the main question of conditioning effects are the effects of group. Although the MANOVA revealed no overall group effect, there was a significant interaction of group and interval [Wilks's lambda $=0.179, F(40,52)=1.775, p<.026]$.

The data were further analyzed by individual MANOVAs at each of the scoring intervals to discover the location 
of the group effects. These analyses revealed a significant group effect during the prenaloxone interval [Wilks's lambda $=0.273, F(22,70)=2.834, p<.0005]$. Neither postnaloxone interval revealed significant group effects [both Wilks's lambda $>0.456, F(22,70)$ s $<$ $1.54, p s>.09$ ].

The sources of the highly significant group effect during the initial recording interval were determined by oneway ANOVAs on each individual measure. Differences were tested by Newman-Keul's comparisons when significant group effects were detected. The results obtained, the mean scores $( \pm S E M)$, and the significance of group differences on each measure are summarized in Table 1.

These comparisons strongly confirm a pattern of enhanced withdrawal in the paired group relative to the other two conditions. Five of the 11 withdrawal measures showed significant differences between the paired and unpaired groups. In addition, 5 measures showed significant differences between the paired and the control groups. In each case, the paired group exhibited more signs of withdrawal. As shown in Table 1, the measures of withdrawal that revealed significant differences indicative of conditioned withdrawal were the reduction in rearing and several measures that involved shaking or tremors. It is important to note that the measures that differentiated the paired group are the ones most frequently reported as reliable indicators of precipitated withdrawal (cf. Blasig et al., 1973; Mucha et al., 1979; Wei, 1973).

By contrast, there is no evidence of withdrawal in the unpaired group. The unpaired group differed significantly from the control group on only two measures, darting and chewing; in both cases, the number of withdrawal signs was lower in unpaired animals. Although the source of this difference is unclear, it is evident by the absence of even a hint of withdrawal in the unpaired group that prior morphine experience alone did not cause the withdrawal signs found in the paired group.

\section{Etonitazene Self-Administration}

The number of responses and reinforcements for each of the groups was analyzed during the six sessions of the etonitazene acquisition phase by separate mixed design ANOVAs with group as the between factor and session as the within factor. An analysis of responses showed a significant overall group effect $[F(2,40)=4.14, p<$ $.05]$, a significant session effect $[F(5,200)=7.57, p<$ $.0001]$, and a nonsignificant session $\times$ group interaction $[F(10,200)=1.51, p>.1]$. The pattern indicated by these effects is shown in Figure 1. The data indicate an overall reduction in responding as a function of repeated sessions. More importantly, they indicate a significant effect of prior morphine experience on etonitazene acquisition. Newman-Keul's comparisons confirm that both morphine-experienced groups of animals responded more for etonitazene than did the saline control group $(p s<$ .5 ). The two morphine groups, however, did not differ from one another.
The analysis of reinforcement during etonitazene acquisition reveals a similar pattern. There was a significant group effect $[F(2,40)=6.43, p<.005]$, a marginal session effect $[F(5,200)=2.03, p<.08]$, and no session $\times$ group interaction $[F(10,200)=1.36, p>.2]$. Again, Newman-Keul's comparisons confirmed that morphineexperienced animals received more etonitazene reinforcements than did saline control animals $(p s<.5)$ but did not differ according to the conditioning manipulation.

\section{DISCUSSION}

The present study provides the first independent replication of conditioned withdrawal under controlled laboratory conditions. The results of this study confirm those of Kelsey and associates (Falls \& Kelsey, 1989; Kelsey et al., 1990) and confirm Siegel's $(1979,1983)$ prediction that conditioned withdrawal can be elicited by signals for opiate administration. This finding is also consistent with much data from human addict populations that show that withdrawal symptoms can be stimulated by natural signals of opiate administration (O'Brien, 1975; O'Brien \& Ternes, 1978; O'Brien, Testa, O'Brien, Brady, \& Wells, 1977; Sideroff \& Jarvik, 1980; Teasdale, 1973; for a review of conditioned-withdrawal studies with human subjects, see O'Brien, Ehrman, \& Ternes, 1986).

Conditioned withdrawal is revealed by the greater incidence of common withdrawal signs in paired animals than in either drug-naive animals or unpaired animals. The absence of any evidence of withdrawal in unpaired animals not only shows that drug experience alone is insufficient to produce the withdrawal seen in the present study but also shows that environmental cues paired with greater levels of abstinence (i.e., $24 \mathrm{~h}$ after morphine) did not stimulate withdrawal signs.

The nature of effective procedures for observing conditioned withdrawal are consistent with Siegel's (1979, 1983) suggestion that drug administration itself is an effective conditioning agent for establishing conditioned withdrawal. The procedure of this study is more similar to that of Kelsey and associates (Falls \& Kelsey, 1989; Kelsey et al., 1990) than to those in studies (Sobrero \& Bouton, 1989; Zellner et al., 1984) in which attempts to observe conditioned withdrawal were unsuccessful. Moreover, the method of opiate administration (s.c. morphine injection) and the temporal parameters of the environmental CS $(50 \mathrm{~min})$ were similar to procedures that effectively produce conditioned compensatory responses using other measures such as analgesia (Krank, 1987; Krank et al., 1981). Although not conclusive, the fact that successful demonstrations of conditioned withdrawal occur with parameters similar to those used in successful conditioned tolerance studies is consistent with Siegel's $(1979,1983)$ characterization of conditioned withdrawal as a conditioned drug-preparatory syndrome related to drug tolerance. 
A.

\section{Responses}

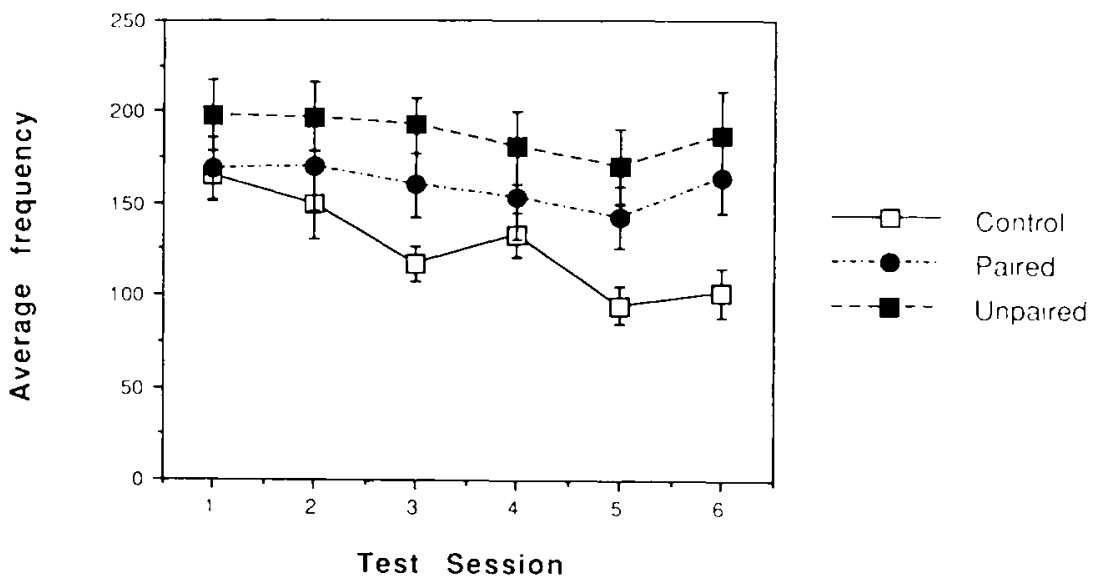

B.

Reinforcements

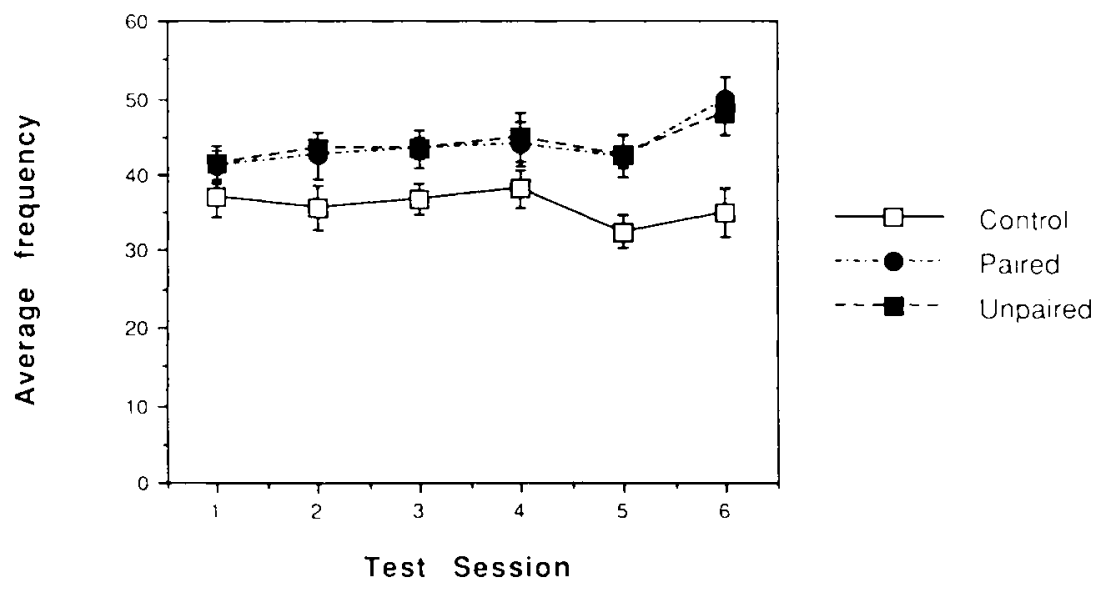

Figure 1. (A) The average barpressing responses of the three groups during the six sessions of testing with sweetened etonitazene as the reinforcement. (B) The reinforcements obtained during the etonitazene test phase.

These results also demonstrate that naloxone administration is not necessary to observe conditioned withdrawal. Withdrawal behavior was observed only prior to naloxone administration. After naloxone administration, there was a general reduction in behavior and no reliable evidence for withdrawal. The inability of naloxone to precipitate withdrawal in this procedure may not be too surprising. Antagonist-precipitated withdrawal occurs when opiate stimulation (usually chronic) is removed by blocking the opiate receptor site. Because withdrawal testing in the present study took place 4 days after the last morphine experience, very little active drug would be displaced.

Although this study provides strong support for conditioned withdrawal, it fails to confirm the expected motivational impact of drug conditioning. Conditioned withdrawal does not enhance the acquisition of opiate selfadministration. Prior morphine experience does enhance operant behavior for the oral solution of etonitazene, but conditioning with morphine has no effect on this behavior. Not only does the lack of a conditioning effect on the acquisition of etonitazene self-administration behavior fail to support a motivational role for conditioned withdrawal (Siegel, 1979; Hinson \& Siegel, 1980), but it also fails to support a motivational role for conditioned incentive (Stewart et al., 1984) and conditioned selective tolerance (Krank, 1989).

The absence of a conditioning effect on opiate consumption in this study is surprising given the work of Hinson et al. (1986), who found that opiate signals do enhance opiate consumption. Moreover, the present study used procedures identical to those used by Krank (1989; Krank et al., 1989) to demonstrate that signals for ethanol enhance the acquisition of operant behavior for, and consumption of, ethanol. Given the sensitivity of the selfadministration test to prior, noncontingent, morphine administration found in this experiment and the sensitivity 
to conditioning with ethanol found in previous studies, it is unlikely that the inability to observe a conditioning effect is due to the use of insensitive procedures.

The main difference between the present study and the successful demonstration of a conditioning effect on opiate consumption (Hinson et al., 1986) was the level of experience of opiate consumption prior to testing. As in our previous work with ethanol, the animals used in this study were drinking the opiate for the first time during the test phase. In contrast, Hinson et al.'s animals had an extensive prior history of opiate consumption.

Evidence from studies of conditioned tolerance to the aversive effects of drugs may help explain the possible contribution of drug-taking history to the present results. The initial acquisition of oral drug-taking behavior is, in part, governed by tolerance to drug effects that lead to taste aversions (e.g., Cappell, Leblanc, \& Endrenyi, 1973). The reduction in self-administration of etonitazene in the drug-naive control group of the present study illustrates a probable taste-aversion effect. Indeed, the effect of prior morphine experience may be best characterized as tolerance to this taste aversion. Both groups show resistance to the reductions seen in the control group.

Several studies have documented that opiate-induced taste aversions are attenuated by prior opiate experience, but unlike some other forms of opiate tolerance, this effect is not based on conditioning with environmental cues (Domjan \& Siegel, 1983). Thus, the taste-aversion impact on etonitazene self-administration would be affected by prior morphine experience but not by the conditioning manipulation. In contrast, tolerance to the taste-aversion properties of ethanol are affected by conditioning (Dacanay \& Riley, 1982). This interpretation is consistent with the finding that ethanol conditioning does affect the acquisition of ethanol self-administration using very similar procedures (Krank, 1989; Krank et al., 1989).

The question remains, does conditioned withdrawal contribute to opiate self-administration in animals with extensive opiate experience? The data of Thompson and Ostlund (1965) and Hinson et al. (1986) suggest that conditioning factors influence opiate self-administration in tolerant and dependent animals. O'Brien et al. (1986) suggest that human opiate use is characterized by different phases associated with different motivations. Relief from withdrawal becomes a motive only in later phases of addiction associated with tolerance and dependence. This line of argument leads to the prediction that conditioned withdrawal may influence opiate consumption only in dependent animals that have learned to gain relief from withdrawal by consuming opiates. The present study confirms that withdrawal can be conditioned with morphine as the US but fails to demonstrate that such withdrawal has motivational consequences.

\section{REFERENCES}

Baker, T. B., \& TIFfany, S. T. (1985). Morphine tolerance as habituation. Psychological Review, 92, 78-108.
Blasig, J., Herz, A., \& Reingold, K. (1973). Development of physical dependence on morphine in respect to time and dosage quantification of the precipitated withdrawal syndrome in rats. Psychopharmacologia, 33, 19-38.

Cappell, H., Leblanc, A. E., Endrenyi, L. (1973). Aversive conditioning by psychoactive drugs: Effects of morphine, alcohol, and chlordiazepoxide. Psychopharmacologia, 29, 239-246.

Carroll, M. E., \& Meisch, R. A. (1979). Concurrent etonitazene and water intake in rats: Role of taste, olfaction, and auditory stimuli. Psychopharmacology, 64, 1-7.

Dacanay, R. J., \& RILEY, A. L. (1982). The UCS preexposure effect in taste aversion learning: Tolerance and blocking are drug specific. Animal Learning \& Behavior, 10, 91-96.

DE WIT, H., \& STEWART, J. (1981). Reinstatement of cocaine-reinforced responding in the rat. Psychopharmacology, 79, 134-143.

DOMJAN, M., \& SIEGEL, S. (1983). Attenuation of the aversive and analgesic effects of morphine by repeated administration: Different mechanisms. Physiological Psychology, 11, 155-158.

Eikelboom, R., \& StewarT, J. (1982). Conditioning of drug-induced physiological responses. Psychological Review, 89, 507-528.

Falls, W. A., \& Kelsey, J. E. (1989). Procedures that produce contextspecific tolerance to morphine in rats also produce context-specific withdrawal. Behavioral Neuroscience, 103, 842-849.

Hinson, R. E., Poulos, C. X., Thomas, W., \& Cappell, H. (1986). Pavlovian conditioning and addictive behavior: Relapse to oral selfadministration of morphine. Behavioral Neuroscience, 100, 368-375.

Hinson, R. E., Siegel, S. (1980). The contribution of Pavlovian conditioning to ethanol tolerance and dependence. In $\mathbf{H}$. Rigter \& J. C. Crabbe (Eds.), Alcohol tolerance, dependence \& addiction (pp. 181. 199). Amsterdam: Elsevier/North-Holland Biomedical.

Kelsey, J. E., Aranow, J. S., \& Mathews, R. T. (1990). Contextspecific morphine withdrawal in rats: Duration and effects of clonidine. Behavioral Neuroscience, 104, 725-733.

Krank, M. D. (1987). Conditioned hyperalgesia depends on the pain sensitivity measure. Behavioral Neuroscience, 101, 854-857.

KRANK, M. D. (1989). Environmental signals for ethanol enhance freechoice ethanol consumption. Behavioral Neuroscience, 103, 365-372.

Krank, M. D., Hinson, R. E., \& SiegeL, S. (1981). Conditioned hyperalgesia is elicited by environmental signals of morphine. Behavioral \& Neural Biology, 32, 148-157.

Krank, M. D., O'Neill, S., \& Brake, L. (1989). The effects of contextual and discrete cues for ethanol on operant responding for ethanol: Support for operant-Pavlovian interactions in drug-taking behavior. Alcoholism: Clinical \& Experimental Research, 13, 302.

Krank, M. D., \& Wall, A. M. (1990). Cue exposure during a period of abstinence reduces the resumption of operant behavior for oral ethanol reinforcement. Behavioral Neuroscience, 104, 725-733.

Mucha, R. F., Kalant, H., \& Linseman, M. A. (1979). Quantitative relationships among measures of morphine tolerance and physical dependence in the rat. Pharmacology, Biochemistry \& Behavior, 10, 397-405.

O'BRIEN, C. P. (1975). Experimental analysis of conditioning factors in human narcotic addiction. Pharmacological Review, 27, 533-534.

O'Brien, C. P., Ehrman, R. N., \& Ternes, J. W. (1986). Classical conditioning in human opioid dependence. In S. R. Goldberg \& I. P. Stolerman (Eds.), Behavioral analysis of drug dependence (pp. 329356). Orlando, FL: Academic Press.

O'Brien, C. P., \& Ternes, J. W. (1978). Conditioning as a cause of relapse in narcotic addiction. In E. Gottheil \& A. T. McLellan (Eds.), Addiction research and treatment: Converging trends. New York: Pergamon.

O’Brien, C. P., Testa, T., O'Brien, T. J., Brady, J. P., \& Wells, B. (1977). Conditioned narcotic withdrawal in humans. Science, 195, 1000-1002.

Poulos, C. X., Hinson, R. E., \& Siegel, S. (1981). The role of Pavlovian conditioning in drug tolerance and dependence: Implications for treatment. Addictive Behaviors, 6, 205-211.

Sideroff, S., \& JARVIK, M. E. (1980). Conditioned responses to videotape showing heroin-related stimuli. International Journal of Addictions, 15, 529-536. 
Siegel, S. (1979). The role of conditioning in drug tolerance and addiction. In J. D. Keehn (Ed.), Psychopharmacology in animals: Research and treatment implications (pp. 143-168). New York: Academic Press.

SIEGEL, S. (1983). Classical conditioning, drug tolerance, and drug dependence. In F. B. G. Y. Israel (Ed.), Research advances in alcohol and drug problems (Vol. 7, pp. 207-246). New York: Plenum.

SiEgEL, S. (1989). Pharmacological conditioning and drug effects. In A. J. Goudie \& M. Emmett-Oglesby (Eds.), Psychoactive drugs (pp. 115-180). Clifton, NJ: Humana Press.

Siegel, S., Hinson, R. E., \& Krank, M. D. (1978). The role of predrug signals in morphine analgesic tolerance: Support for a Pavlovian conditioning model of tolerance. Journal of Experimental Psychology: Animal Behavioral Processes, 4, 188-196.

Siegel, S., Krank, M. D., \& Hinson, R. E. (1987). Anticipation of pharmacological and nonpharmacological events: Classical conditioning and addictive behavior. Journal of Drug Issues, 17, 83-110.

Sobrero, A. P., \& Bouton, M. E. (1989). Effects of stimuli present during oral morphine administration on withdrawal and subsequent consumption. Psychobiology, 17, 179-190.

Stewart, J., de WIT, H., EIKelboom, R. (1984). Role of unconditioned and conditioned drug effects in the self-administration of opiates and stimulants. Psychological Review, 91, 251-268.

TABakoff, B., Hoffman, P. L. (1988). Tolerance and the etiology of alcoholism: Hypothesis and mechanism. Alcoholism: Clinical \& Experimental Research, 12, 184-186.
Teasdale, J. (1973). Conditioned abstinence in narcotic addicts. International Journal of Addictions, 8, 273-292.

Thompson, T., \& OSTLUND, J. W. (1965). Susceptibility to readdiction as a function of the addiction and withdrawal environments. Journal of Comparative \& Physiological Psychology, 60, 388-392.

Tiffany, S. T., Petrie, E. C., Baker, T. B., Dahl, J. L. (1983). Conditioned morphine tolerance in the rat: Absence of a compensatory response and cross-tolerance with stress. Behavioral Neuroscience, 97, 335-353.

Trapold, M. A., \& Overmier, J. B. (1972). The second leaming process in instrumental conditioning. In A. H. Black \& W. F. Prokasy (Eds.), Classical conditioning: II. Current theory and research (pp. 427-452). New York: Appleton-Century-Crofts.

WEI, E. (1973). Assessment of precipitated abstinence in morphinedependent rats. Psychopharmacologia, 28, 35-44.

WIKLER, A. (1973). Conditioning of successive adaptive responses to the initial effects of drugs. Conditional Reflex, 8, 193-210.

Wikler, A., Martin, W. R., Pescor, F. T., \& Eades, C. G. (1963). Factors regulating oral consumption of an opioid (etonitazine) by morphine addicted rats. Psychopharmacologia, 5, 55-76.

Zellner, D. A., DaCanay, R. J., \& Riley, A. L. (1984). Opiate withdrawal: The result of conditioning or physiological mechanisms? Pharmacology, Biochemistry \& Behavior. 20, 175-180.

(Manuscript received October 28, 1992 ; revision accepted for publication March 11, 1993.) 